\title{
A Study on Performance Analysis of V2V Communication Based AEB System Considering Road Friction at Slopes
}

\author{
Sangduck Jeon ${ }^{1}$, Jungeun Lee ${ }^{1}$ and Byeongwoo Kim ${ }^{2}$ \\ ${ }^{1}$ Graduate School of Electrical Engineering, University of Ulsan, \\ 93 Daehak-ro, Ulsan, Republic of Korea \\ jsd0831@gmail.com,rotnn429@gmail.com \\ ${ }^{2}$ School of Electrical Engineering, University of Ulsan, \\ 93 Daehak-ro, Ulsan, Republic of Korea \\ Corresponding Author: bywokim@ulsan.ac.kr
}

\begin{abstract}
An autonomous emergency braking (AEB) system based on Vehicle to Vehicle (V2V) communication considering road friction at slopes was proposed in this study. Conventional AEB systems have limited performance of effective braking according to road conditions because they mostly consider only particular road conditions on straight roads. To solve this limitation, a new control logic was proposed to calculate the braking timing by considering road condition at slopes. The TTC (time to collision) method, which is the collision risk, was introduced in the proposed AEB system based on information of the host vehicle and a nearby vehicle. To verify the proposed AEB system, various road conditions, vehicle speeds and slopes were reflected in this scenario. The AEB system proposed through this analysis showed enhanced braking and collision avoidance performances compared to conventional AEB systems.
\end{abstract}

Keywords: AEB (Autonomous Emergency Braking), TTC (Time to Collision), Road condition, gradient, V2V (Vehicle to Vehicle), V2I (Vehicle to Infra)

\section{Introduction}

According to the 2014 traffic accident data analyzed by the domestic National Police Agency, ap-proximately $73 \%$ of vehicular accidents occur because of the carelessness of drivers [1]. Thus, acci-dents that occur by late braking timing owing to carelessness such as distraction and dozing off while driving make up a large part of the total number of accidents. As interest in car safety has increased, research on advanced driver assistant systems (ADAS) is being actively conducted in the automobile market, to prevent accidents due to the carelessness of drivers [2]. Among several functions in the ADAS, the AEB system analyzes the collision risk with the vehicle ahead and prevents or avoids accidents by automatic braking in dangerous situations [3]. AEB systems generally detect danger through obstacle detecting sensors such as cameras, LIDAR (light detection and ranging), and radar (radio detecting and ranging) [4]. Obstacle detecting sensors such as cameras and radars can only detect obstacles right in front of the vehicle, because of the physical interference of nearby vehicles. Owing to this limitation, a dead zone occurs on a slope. When a dead zone occurs on a slope by blocking obstacles such as a vehicle, normal collision avoidance becomes difficult [5]. AEB systems that integrate Vehicle to Vehicle (V2V) and Vehicle to Infra (V2I) are being proposed to reduce the accident rate because of this problem [6].

The Euro NCAP AEB evaluation procedure is generally followed to evaluate automobile AEB sys-tems [7]. Standardized road conditions (dry asphalt) are only reflected in the Euro NCAP AEB evaluation procedure. 
Road surface friction is a road feature that has significant meaning in road or driving information that drivers recognize and that can be effectively used in the control systems of vehicles. When only particular road conditions are reflected, limited braking and collision performance can be realized, because frequently changing road conditions cannot be satisfied [8]. Research on the AEB system evaluation method in a particular road condition (dry asphalt) was performed in existing research [9]. The conventional AEB system used the relative velocity and relative distance between the target vehicle and host vehicle to calculate the time to collision (TTC). Other features that affect braking were excluded in this type of system because only the relative velocity and relative distance at the braking timing to activate the brakes were used [10]. Therefore, further research is required to solve the limits of sensor detection zones and to enhance AEB system performance through decisions about collision risks in various road condition environments.

Thus, a plan to enhance AEB systems considering road condition was presented by integrating $\mathrm{V} 2 \mathrm{~V}$ communication technology to overcome these problems such as dead zones and obstacles such as vehicles. Additionally, the conventional sensor-based AEB system was used to evaluate its usabil-ity in comparison with the proposed AEB system.

\section{AEB System Design}

\subsection{V2V Communication Based AEB System}

Figure 1 shows the block diagram of the AEB system proposed in this paper. A vehicle model that can implement road conditions and specific dynamic characteristics is required to design the proposed AEB system. A vehicle model with road conditions and high degree of freedom was used by using CarSim. PreScan was used to configure the V2V communication environment and driving environment. The DNPW algorithm was designed, and PreScan and CarSim were interfaced using MATLAB/Simulink.

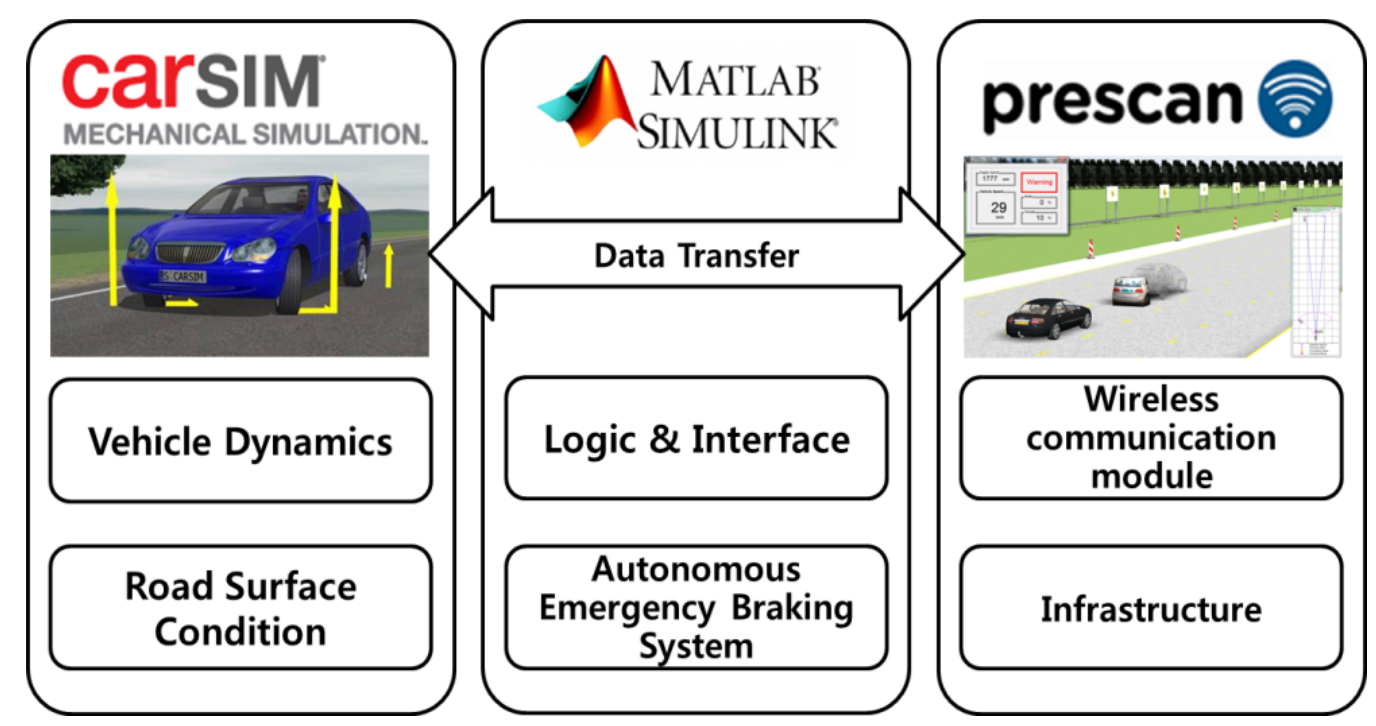

Figure 1. AEB System Block Diagram

Figure 2 shows the proposed AEB system block that considers the road condition. The proposed AEB system is mainly composed of two parts. The first part calculates the braking timing by considering road conditions, and the second part calculates collision risk according to road shape for effective braking on various roads. 


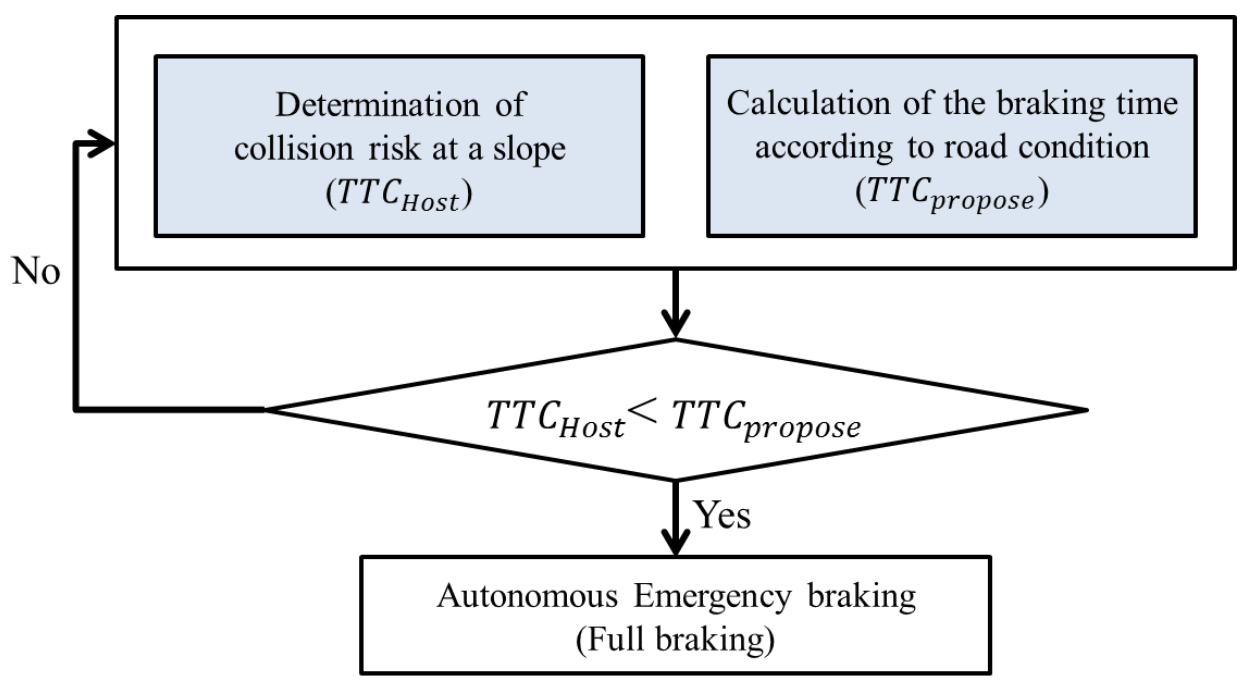

Figure 2. AEB System Flow Chart

When the proposed AEB system assesses the collision status, maximum braking is applied at the instant when the TTC $\left(T T C_{\text {Host }}\right)$, the time required until the collision point, becomes smaller than the braking timing (TTC $\left.C_{\text {propose }}\right)$ according to the road condition, as shown in Equation (1).

$$
T T C_{\text {Host }}<T T C_{\text {propose }} \text { (Full braking) }
$$

\subsection{Calculation of Braking Timing According to Road Conditions}

An AEB system causes a vehicle to autonomously activate the brake when assessing a dangerous situation by analyzing the collision risk with the vehicle ahead. However, quicker braking is required because friction between the tire and road surface decreases, such as when the road is wet or frozen. Therefore, an algorithm that calculates the optimum timing of braking intervention according to road conditions is proposed in this paper.

As shown in Figure 3, the effect of a slope on a vehicle must be considered to safely avoid collision on slopes.

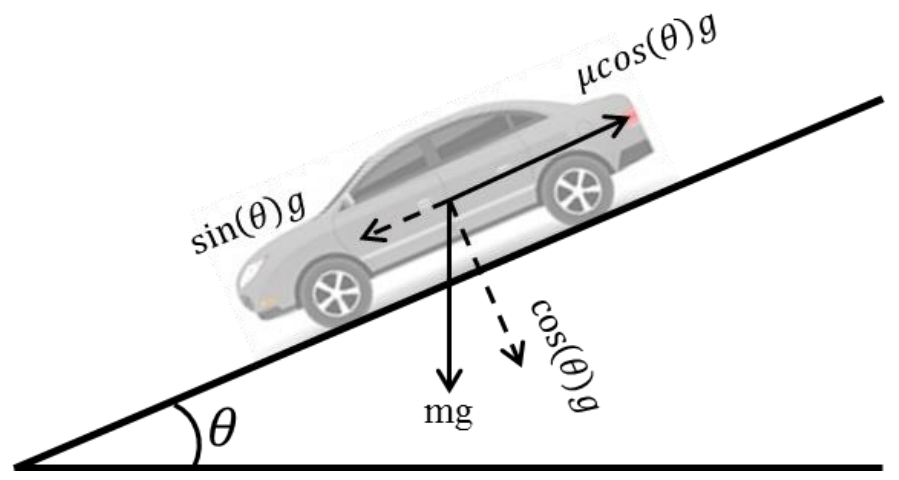

Figure 3. Effect According to Gradient

Braking timing (TTC $\left.C_{\text {propose }}\right)$ according to the road condition can be found by using the maximum deceleration according to the road condition and gradient. The deceleration movement of the vehicle is assumed as the uniform deceleration movement to calculate maximum deceleration $\left(a_{\mu}\right)$, time until stopping $\left(T_{\mu}\right)$, and maximum deceleration distance 
$\left(S_{\mu}\right)$. Maximum deceleration can be found by Equation (3) in which $\mu$ is the friction coefficient, $g$ is the acceleration of gravity, and $\theta$ is the gradient. Gradient is calculated by using DGPS data as shown in Equation (2). Time until stopping can be calculated as shown in Equation (4). Distance until stopping can be calculated as shown in Equation (5). The collision risk index ( $\left.T T C_{\text {propose }}\right)$ at a slope is calculated using the deducted maximum deceleration and maximum deceleration distance, as shown in Equation (6).

$$
\begin{gathered}
\theta=\tan ^{-1}\left(v_{z G P S} / \sqrt{v_{x G P S^{2}}+v_{y G P S}{ }^{2}}\right) \\
a_{\mu}=-\mu \cos (\theta)+g \sin (\theta) \\
T_{\mu}=\frac{V_{\text {Host }}}{a_{\mu}} \\
S_{\mu}=\frac{V_{\text {Host }} \times T_{\mu}}{2} \\
T T C_{\text {propose }}=\frac{s_{\mu}}{V_{\text {Host }}}
\end{gathered}
$$

\subsection{Decision of Collision Risk at Slope}

The method of calculating collision risk by using the relative distance of each vehicle is not suitable at a slope. As shown in Figure 4, accurate relative distance cannot be found because the absolute location value of the vehicle is received through DGPS. Therefore, slope distance was used instead of relative distance of a vehicle in this study, as shown in Equation (7). The collision risk is found by using the relative distance at a slope as shown in Equation (8).

$$
\begin{array}{r}
S_{\text {Slope }}=\frac{\text { Relative distance }}{\cos (\theta)} \\
T T C_{\text {Host }}(s)=\frac{S_{\text {slope }}(m)}{\text { Relative velocity }(m / s)}
\end{array}
$$

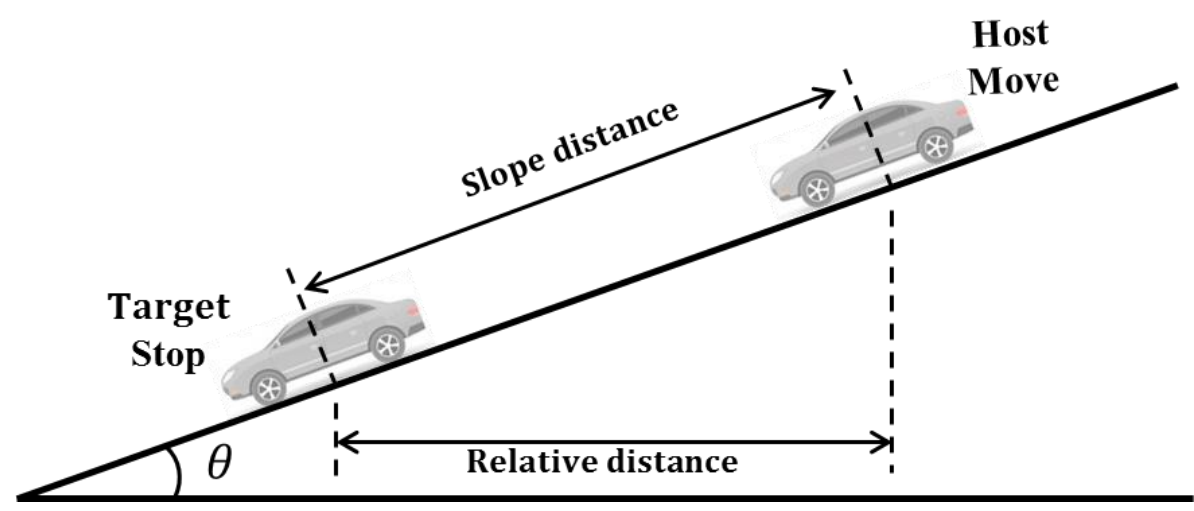

Figure 4. Relative Distance at Slope (Slope Distance)

\section{Verification of Algorithm}

\subsection{Simulation Configuration}

Euro NCAP scenarios were applied in the slope scenarios. There are three types of Car to Car scenarios in Euro NCAP. They are classified into CCRs, CCRm, and CCRb scenarios according to the velocities of the host vehicles and target vehicle. 
As shown in Figure 4, the moving condition of vehicle in the CCRs scenario, in which the host vehicle moves and the target vehicle stops, is applied. The conventional AEB system and proposed AEB system were comparatively analyzed by changing velocity, gradient, and road friction in slopes.

Scenario conditions were presented to compare the simulation results of the conventional AEB sys-tem and proposed AEB system in various situations.

\subsection{Simulation Results}

Table 1 and Table 2 show the simulation results of collision status between vehicles according to velocity and road condition on slopes. The collision avoidance rate of the conventional AEB system in 4 [\%] gradient conditions is 16.7[\%] as shown in Table 1. Collision in all scenarios except dry asphalt and wet asphalt was observed at $40[\mathrm{~km} / \mathrm{h}]$. The collision avoidance rate of the proposed AEB system is 100[\%]. Avoidance in all cases was observed.

As shown in Table 2, the collision avoidance rate of the conventional AEB system in $10[\%]$ gradi-ent condition is 0[\%]. Collision was observed in all scenarios. However, the collision avoidance rate of the proposed AEB system is 100[\%]. Avoidance in all presented velocities and road conditions was confirmed.

Table 1. Collision Status According to Velocity and Road Condition in 4[\%] Gradient Condition

\begin{tabular}{|c|c|ccc|}
\hline \multirow{2}{*}{ AEB System } & $\begin{array}{r}\text { Road } \\
\text { Cendition }\end{array}$ & Dry asphalt & Wet asphalt & Snow asphalt \\
& Velocity & Avoidance & Avoidance & Collision \\
& 40 & Avoidance & Collision & Collision \\
& 60 & Collision & Collision & Collision \\
& 80 & Collision & Collision & Collision \\
\hline \multirow{3}{*}{ Proposed } & 100 & Avoidance & Avoidance & Avoidance \\
& 40 & Avoidance & Avoidance & Avoidance \\
& 60 & Avoidance & Avoidance & Avoidance \\
& 100 & Avoidance & Avoidance & Avoidance \\
\hline
\end{tabular}


Table 2. Collision Status According to Velocity and Road Condition in 10[\%] Gradient Condition

\begin{tabular}{|c|c|c|c|c|}
\hline AEB System & $\begin{array}{l}\text { Road } \\
\text { Velocity }\end{array}$ & Dry asphalt & Wet asphalt & Snow asphalt \\
\hline \multirow{4}{*}{ Conventional } & 40 & Collision & Collision & Collision \\
\hline & 60 & Collision & Collision & Collision \\
\hline & 80 & Collision & Collision & Collision \\
\hline & 100 & Collision & Collision & Collision \\
\hline \multirow{4}{*}{ Proposed } & 40 & Avoidance & Avoidance & Avoidance \\
\hline & 60 & Avoidance & Avoidance & Avoidance \\
\hline & 80 & Avoidance & Avoidance & Avoidance \\
\hline & 100 & Avoidance & Avoidance & Avoidance \\
\hline
\end{tabular}

Among the simulation results of the conventional AEB system and proposed AEB system shown according to scenarios, two main simulation results were shown in this paper. Through simulation results under the same condition, the conventional AEB system and proposed AEB system were comparatively analyzed.

Figure 5 and Figure 6 show the main simulation results of the conventional AEB system in $4[\%]$ gradients. The scenario has dry asphalt road condition and $60[\mathrm{~km} / \mathrm{h}]$ velocities.

Figure 5 shows the graph of TTC and Figure 6 shows the graph of host vehicle acceleration. It was confirmed that the braking timing is identical despite a changing road condition and gradient. Partial braking was applied when TTC was 1.6[s] and full braking was applied when TTC was $0.6[\mathrm{~s}]$. The host vehicle collided with the target vehicle because braking did not completely take place, and TTC was 0 . Braking force of $0.37[\mathrm{~g}]$ was applied when TTC was $1.6[\mathrm{~s}]$, and braking force of $0.73[\mathrm{~g}]$ was applied when TTC was $0.6[\mathrm{~s}]$. Maximum deceleration was lesser than $0.85 \mathrm{~g}$ despite dry asphalt road condition. This is because the gradient affected braking.

Figure 7 and Figure 8 show the main simulation results of the proposed AEB system in 4 [\%] gradients. The scenario has dry asphalt road condition and $60[\mathrm{~km} / \mathrm{h}]$ velocities.

Figure 7 shows the graph of TTC, and Figure 8 shows the graph of host vehicle acceleration. TCC larger than 0 is shown beyond $22.5[\mathrm{~s}]$ and relative distance is $0.2[\mathrm{~m}]$. This means that the two vehicles avoided collision. Unlike the conventional AEB system where braking was applied when TTC was $1.6[\mathrm{~s}]$, braking was applied at less than $1.09[\mathrm{~s}]$ of TCC in the proposed AEB system considering the road condition. This information could show that collision was avoided. 


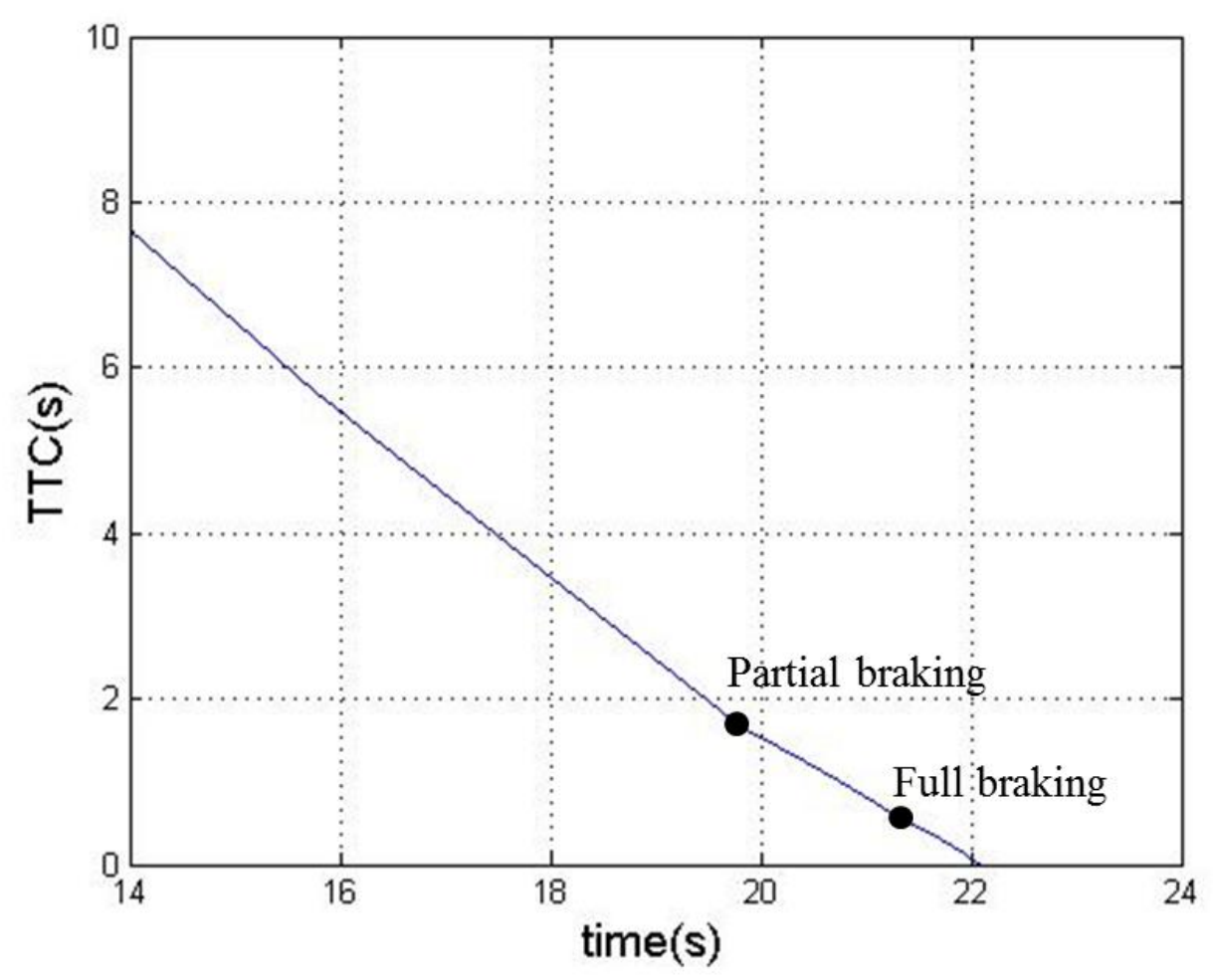

Figure 5. TTC Results of Conventional AEB System (4\%, $60[\mathrm{~km} / \mathrm{h}]$, Dry asphalt)

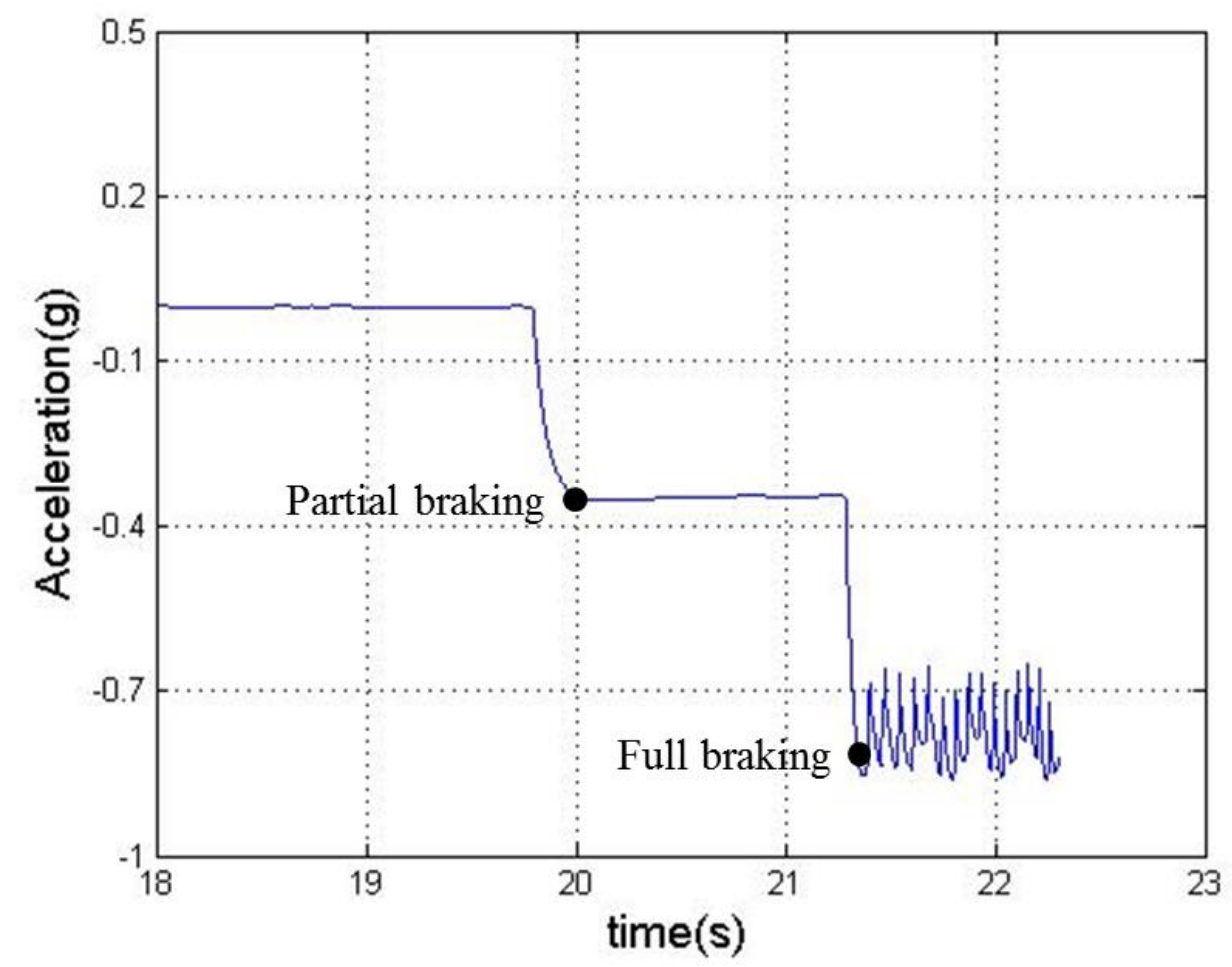

Figure 6. Acceleration Results of Conventional AEB System $(4 \%, 60[\mathrm{~km} / \mathrm{h}]$, Dry asphalt) 


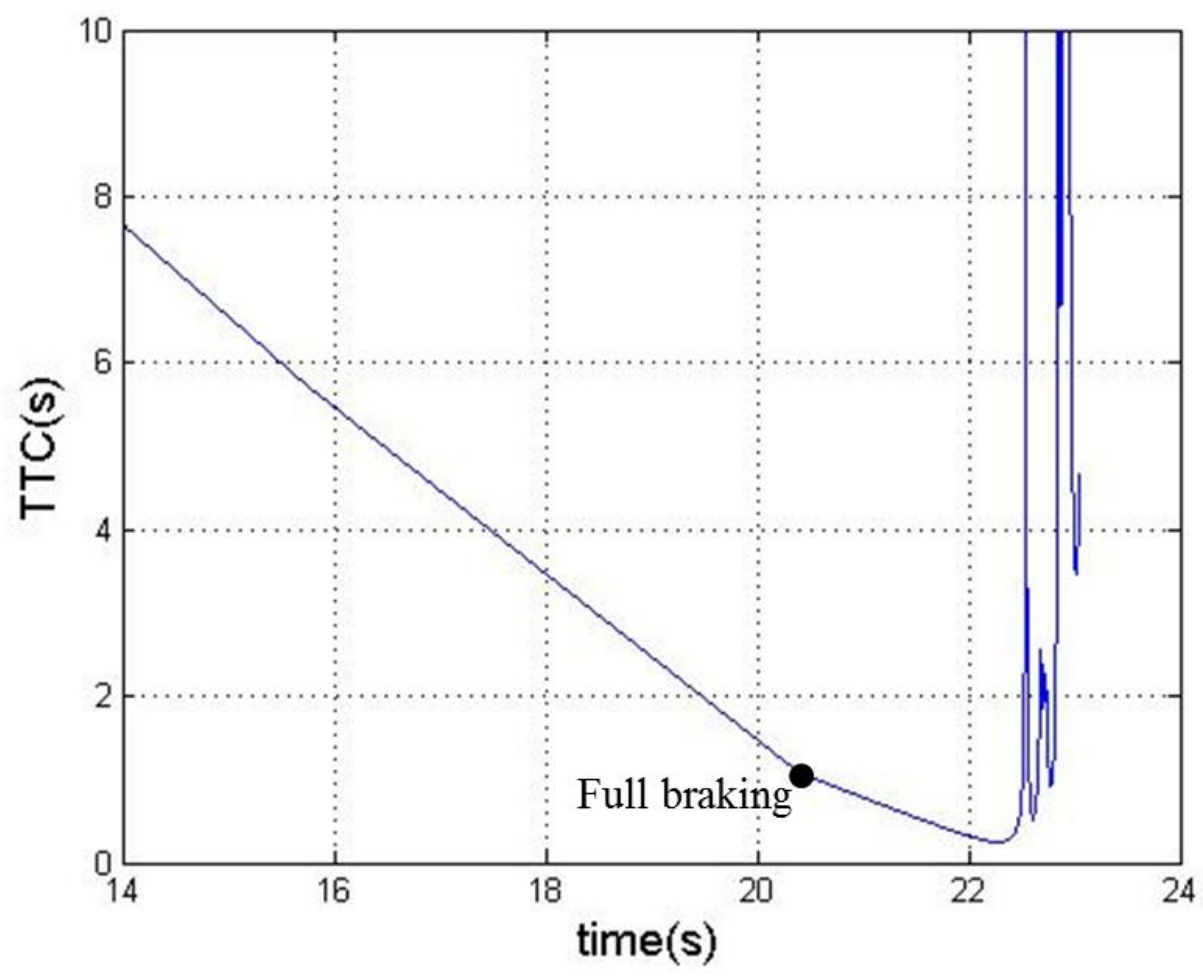

Figure 7. TTC Results of Proposed AEB System (4\%, $60[\mathrm{~km} / \mathrm{h}]$, Dry asphalt)

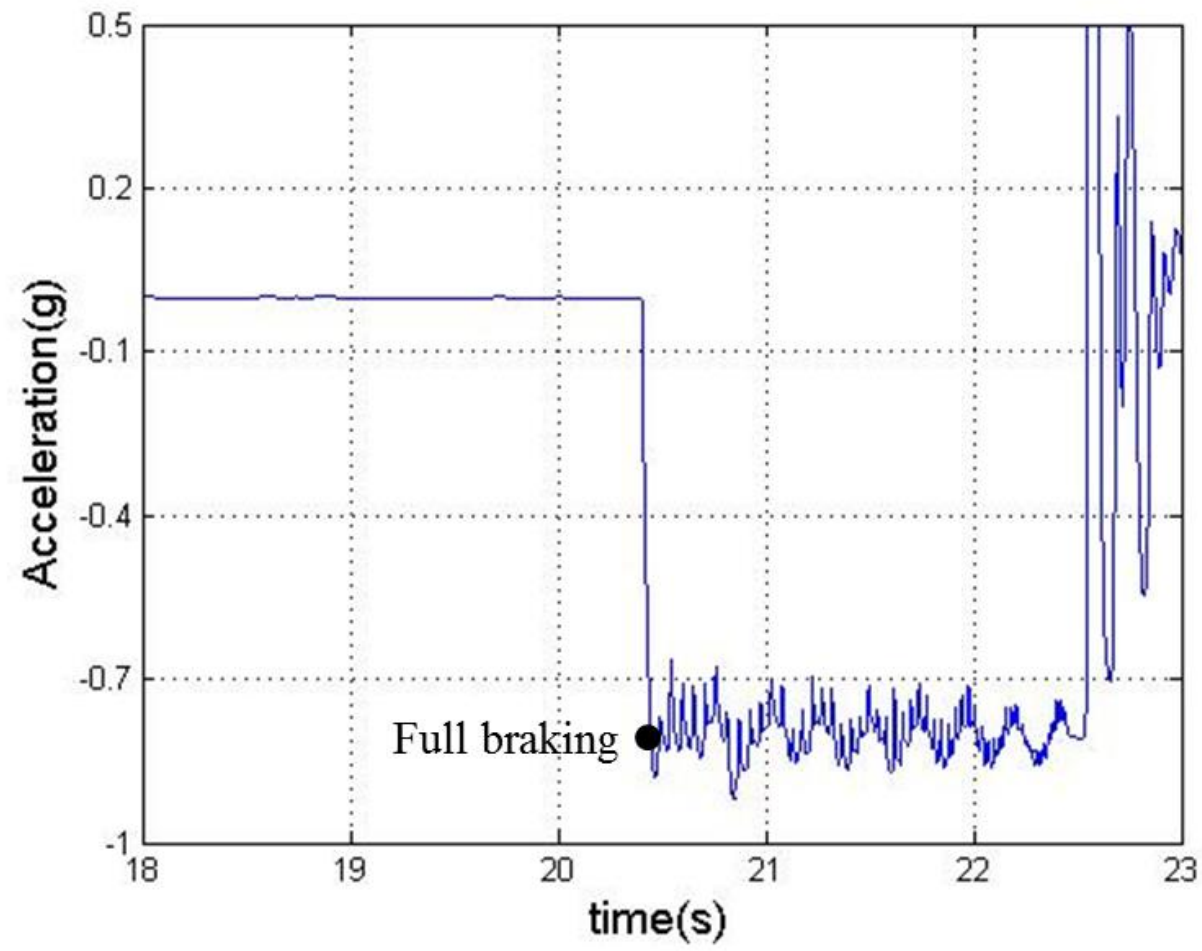

Figure 8. Acceleration Results of the Proposed AEB System $(4 \%, 60[\mathrm{~km} / \mathrm{h}]$, Dry asphalt) 


\section{Conclusions}

In this paper, research on the enhancement of AEB performance considering road conditions were conducted based on $\mathrm{V} 2 \mathrm{~V}$ communication environment. The proposed AEB system deducted collision risks in each road environment using location and velocity information of the host vehicle and a nearby vehicle based on V2V communication. Further, time required stopping, and the velocity of the host vehicle according to road condition and gradient were used to calculate the braking timing. The operation timing of the proposed AEB system is determined by using the braking timing according to collision risk and road condition on slopes.

Simulation results of the conventional AEB were analyzed to evaluate the usefulness of the pro-posed algorithm. The simulation results of the conventional AEB system and proposed AEB system are as follows: the collision avoidance rate of the conventional AEB system in $4[\%]$ gradients is $16.7[\%]$, and the collision avoidance rate of the proposed AEB system is 100[\%]. In the conventional AEB system, avoidance was observed in $40[\mathrm{~km} / \mathrm{h}]$, dry asphalt, and wet asphalt cases, and collision was observed in the other scenarios. The collision avoidance rate of the conventional AEB system in $10[\%]$ gradient is $0[\%]$, and the collision avoidance rate of the proposed AEB system is 100[\%]. Col-lision was observed in all scenarios in the conventional AEB system. The proposed AEB avoided collision in all scenarios. Through these results, the proposed AEB system was shown to have en-hanced braking performance compared to the conventional system.

An AEB system that considers road conditions based on $\mathrm{V} 2 \mathrm{~V}$ communication environment on slopes was proposed in this paper. The usability was confirmed through comparison with the conven-tional AEB system. Research on collision avoidance systems, according to additional road environ-ments, and research on collision avoidance systems considering driving patterns of drivers are planned to be performed in future research.

uthor names and affiliations are to be centered beneath the title and printed in Times New Roman 12-point, non-boldface type. (See example below)

\section{Acknowledgments}

This work was supported by the IT Research and development program of The MOTIE/KEIT (Ministry of Trade, Industry and Energy/Korea Evaluation Institute of Industrial Technology) [N0000889, The infrastructure build of wireless and application service evaluation - certification for cooperative vehicular safety systems.

The research was supported by the world Class 300 project, "Combination of Octa Band antenna System for Ethernet Communication and safe driving system" (Grant Number: S2343331)

\section{References}

[1] Traffic Accident Analysis System, http://taas.koroad.or.kr/

[2] C. Rommerskirchen, M. Helmbrecht and K. Bengler, "Increasing complexity of driving situations and its impact on an ADAS for anticipatory assistance for the reduction of fuel consumption", IEEE Intelligent Vehicles Symposium, (2013), pp. 573-578

[3] “Automated Emergency Brake Systems: Technical requirements, costs and benefits", Published Project Report, European Commision, (2008).

[4] H. Kim and B. Song, "Vehicle Recognition Based on Radar and Vision Sensor Fusion for Auto-matic Emergency Braking”, 2013 13th International Conference on Control, Automation and Systems, (2013), pp. 1342-1346

[5] H. Cho and B. Kim, "Analysis of Forward collision Warning System Based on Vehicle-mounted Sensors on Roads with an Up-Down Road gradient", Contemporary Engineering Sciences, vol. 7, (2014), pp. 1139-1145 
[6] N. Kaempchen, B. Schiele and K. Dietmayer, "Situation Assessment of an Autonomous Emergency Brake for Arbitrary Vehicle-to-Vehicle Collision Scenarios", IEEE Conference on Intelligent Transportation Systems, (2009), pp. 678-687

[7] Euro NCAP AEB Test Protocol, EURO NCAP, http://www.eruoncap.com, (2015).

[8] R. Heseinnezhad and A. B. Hadiashar, "Efficient Antilock Braking by Direct Maximization of TireRoad Frictions", IEEE Transaction on Industrial Electronics, (2010), pp. 3593-3600

[9] C. Grover, I. Knight, F. Okoro, I. Simmons, G. Couper, P. Massie and B. Smith, "Automated Emergency Brake Systems : Technical requirement, costs and benefits", Published Project Report, (2008).

[10] D. Kristofer and C. Hampton, "Safety Benefits of Forward Collision Warning, Brake Assist, and Autonomous Braking Systems in Rear-End Collisions", IEEE Transactions on Intelligent Transportation Systems, (2012), pp.1546-1555.

\section{Authors}

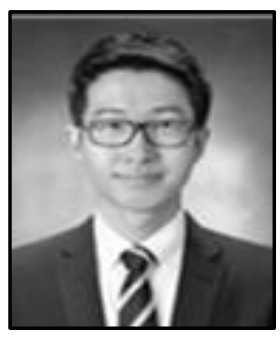

Sangduck Jeon, he received the B.S. Degree in electrical engineering from Ulsan University, Ulsan, South Korea, in 2014. He is currently working toward the M.S. degree in automotive Electronics and control laboratory from Ulsan University, Ulsan, South Korea. His main research activities and interests are autonomous car and autonomous emergency braking (AEB) system.

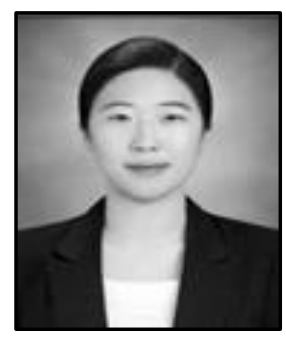

JungEun Lee, she being in attendance at the B.S. Degree in elecrical engineering from Ulsan University, Ulsan, South Korea, from 2013. Her current research interests include advanced driving assistance system (ADAS) and autonomous emergency braking (AEB) system.

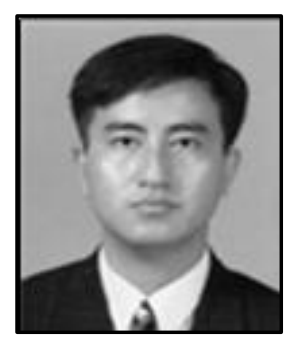

Byeongwoo Kim, he received the B.E, M.E. and Ph.D degree in Precision Mechanical Engineering from Hanyang University. He worked at KOSAKA Research Center in 1989. He worked at KATECH electrical technology Research Center from 1994 to 2006. Now he is a professor in the School of electrical engineering in University if Ulsan, Ulsan, South Korea from 2006. His current research interests include advanced driving assistance system (ADAS), and autonomous emergency braking (AEB) system. 